\title{
Estimating $r_{B}^{D \pi}$ as an input to the determination of the CKM angle $\gamma$
}

\author{
Matthew Kenzie, ${ }^{1}$ Maurizio Martinelli, ${ }^{2}$ and Niels Tuning ${ }^{3}$ \\ ${ }^{1}$ CERN, Geneva, Switzerland \\ ${ }^{2}$ Ecole Polytechnique Fédérale de Lausanne (EPFL), Lausanne, Switzerland \\ ${ }^{3}$ National institute for subatomic physics (Nikhef), Amsterdam, The Netherlands
}

(Received 30 June 2016; published 19 September 2016)

\begin{abstract}
The interference between Cabibbo-favored and Cabibbo-suppressed $B \rightarrow D \pi$ decay amplitudes provides sensitivity to the CKM angle $\gamma$. The relative size of the interfering amplitudes is an important ingredient in the determination of $\gamma$. Using branching fractions from various $B \rightarrow D h$ decays, and the measured value for $r_{B}^{D K}$, the magnitude of the amplitude ratio of $B^{+} \rightarrow D^{0} \pi^{+}$and $B^{+} \rightarrow \bar{D}^{0} \pi^{+}$decays is estimated to be $r_{B}^{D \pi}=0.0053 \pm 0.0007$.
\end{abstract}

DOI: $10.1103 /$ PhysRevD.94.054021

\section{INTRODUCTION}

The CKM description of charged-current quark transitions has been experimentally scrutinized to an impressive accuracy. The CKM angle $\gamma$ encapsulates the relative phase between $b \rightarrow c$ and $b \rightarrow u$ quark transitions, $\gamma \equiv \arg \left[-\frac{V_{u d} V_{u b^{*}}}{V_{c d} V_{c b^{*}}}\right]$, and is determined with a precision of $7^{\circ}$, as compared to a precision below $3^{\circ}$ deduced from indirect measurements [1,2].

The interference between $B^{+} \rightarrow \bar{D}^{0} \pi^{+}$and $B^{+} \rightarrow D^{0} \pi^{+}$ decays, with the $D^{0}$ and $\bar{D}^{0}$ meson decaying to the same final state (charge conjugation is implied throughout), provides sensitivity to the relative weak phase $\gamma$ [3-5]. Experimental determination of $\gamma$ from $B \rightarrow D \pi$ like decays is influenced by the effect of the unknown hadronic parameters: $r_{B}^{D \pi}$, the relative magnitude of the Cabibbosuppressed $B^{+} \rightarrow D^{0} \pi^{+}$amplitude compared to the Cabibbo-favored $B^{+} \rightarrow \bar{D}^{0} \pi^{+}$amplitude, and $\delta_{B}^{D \pi}$, the strong phase difference between the favored and suppressed modes. The ratio of amplitudes, $r_{B}^{D \pi}$, determines the size of the interference effect, and hence the sensitivity to the CKM angle $\gamma$.

A previous simultaneous determination of $\gamma, r_{B}^{D \pi}$ and $\delta_{B}^{D \pi}$ from the LHCb Collaboration, using $B \rightarrow D \pi$ like modes, found multiple solutions for $r_{B}^{D \pi}$ [6]. Consequently, an estimate of its magnitude can provide useful information to improve the determination of $\gamma$ [7].

In this paper we estimate the ratio of amplitudes

$$
r_{B}^{D \pi} \equiv A\left(B^{+} \rightarrow D^{0} \pi^{+}\right) / A\left(B^{+} \rightarrow \bar{D}^{0} \pi^{+}\right)
$$

using branching fractions from various $B \rightarrow D h$ decays, that proceed through similar decay topologies [8], and

Published by the American Physical Society under the terms of the Creative Commons Attribution 3.0 License. Further distribution of this work must maintain attribution to the author(s) and the published article's title, journal citation, and DOI. using the measured value of $r_{B}^{D K}$ [7]. A similar approach was used to estimate the ratio of amplitudes for the decays $B^{0} \rightarrow D^{ \pm} \pi^{\mp}$ [9]. An overview of the decays used is given in Table I. The amplitudes of the decays that involve a kaon in the final state are denoted by primed symbols.

At tree level, the $B^{+} \rightarrow \bar{D}^{0} \pi^{+}$amplitude receives contributions from a color-allowed $(T)$ and color-suppressed topology $(C)$, whereas the $B^{+} \rightarrow D^{0} \pi^{+}$amplitude proceeds predominantly through the color-suppressed topology $\left(C^{u b}\right)$ and also via the annihilation topology, as illustrated in Fig. 1, where the superscript $u b$ indicates that the decay proceeds through a $b \rightarrow u$ transition.

The method to estimate $r_{B}^{D \pi}$ with $B^{0} \rightarrow \bar{D}^{0} K^{0}$ decays is given in Sec. II A, whereas the use of $B^{0} \rightarrow \bar{D}^{0} \pi^{0}$ decays is shown in Sec. II B. The effect of the annihilation diagram is estimated in Sec. II C.

\section{ESTIMATING $r_{B}^{D \pi}$ FROM BRANCHING FRACTIONS}

The expression for the branching fraction takes the following form:

TABLE I. The decays under study are listed, with the topologies contributing to the amplitude, the branching fraction, and the relevant CKM elements. T, $C, E$ and $A$ stand for colorallowed tree, color-suppressed tree, $W$-exchange and annihilation topologies, respectively. The primed symbols indicate the decays with a kaon as the bachelor particle in the final state. The factor $\sqrt{2}$ originates from the isospin decomposition of the neutral pion, $\left|\pi^{0}\right\rangle=(u \bar{u}-d \bar{d}) / \sqrt{2}$.

\begin{tabular}{lccc}
\hline \hline Decay & Topology & $\mathrm{BR}\left(\times 10^{-4}\right)[10]$ & CKM factor \\
\hline$A\left(B^{+} \rightarrow \bar{D}^{0} \pi^{+}\right)$ & $T+C$ & $48.1 \pm 1.5$ & $V_{c b} V_{u d}$ \\
$A\left(B^{0} \rightarrow \bar{D}^{0} \pi^{0}\right)$ & $(C-E) / \sqrt{2}$ & $2.63 \pm 0.14$ & $V_{c b} V_{u d}$ \\
$A\left(B^{+} \rightarrow \bar{D}^{0} K^{+}\right)$ & $T^{\prime}+C^{\prime}$ & $3.70 \pm 0.17$ & $V_{c b} V_{u s}$ \\
$A\left(B^{0} \rightarrow \bar{D}^{0} K^{0}\right)$ & $C^{\prime}$ & $0.52 \pm 0.07$ & $V_{c b} V_{u s}$ \\
$A\left(B^{+} \rightarrow D_{s}^{+} \phi\right)$ & $A^{\prime}$ & $0.017_{-0.007}^{+0.012}$ & $V_{u b} V_{c s}$ \\
\hline \hline
\end{tabular}


(a) $T$ :

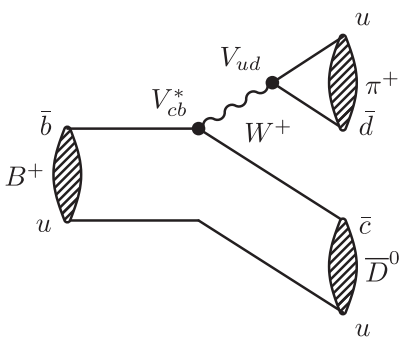

(b) $C$ :


FIG. 1. (a), (b) The color-allowed (tree) ( $T$ ) and colorsuppressed $(C)$ topologies contributing to the $B^{+} \rightarrow \bar{D}^{0} \pi^{+}$ amplitude proceeding through $V_{c b}$, and (c), (d) the colorsuppressed $\left(C^{u b}\right)$ and annihilation topologies contributing to the $B^{+} \rightarrow D^{0} \pi^{+}$amplitude proceeding through $V_{u b}$.

$$
\mathrm{BR}(B \rightarrow D h)=|A(B \rightarrow D h)|^{2} \Phi_{D h}^{d} \tau_{B},
$$

where $h$ is a pion or a kaon, $\Phi_{D h}^{d}$ is a phase-space factor, $A(B \rightarrow D h)$ is the total amplitude (containing the CKM elements, form factors and decay constants) and $\tau_{B}$ is the lifetime of the $B$ meson. Contributions from "rescattering" (like $B^{+} \rightarrow D^{-} \pi^{+} \rightarrow \overline{D^{0}} \pi^{0}$ ) are small, as shown within the framework of QCD factorization by Beneke et al. [11].

The following estimate of the ratio of amplitudes can be made:

$$
\begin{aligned}
r_{B}^{D \pi} & =\frac{A\left(B^{+} \rightarrow D^{0} \pi^{+}\right)}{A\left(B^{+} \rightarrow \bar{D}^{0} \pi^{+}\right)}=\frac{\left|C^{u b}\right|}{|T+C|} \\
& =\left|\frac{V_{u b} V_{c d}}{V_{c b} V_{u d}}\right| \frac{z|C|}{|T+C|}
\end{aligned}
$$

where $z$ quantifies the ratio between the hadronic parts of the two color-suppressed tree diagrams proceeding through a $b \rightarrow c$ or $b \rightarrow u$ transition (shown in Fig. 1), $C^{u b}=z C \times\left(V_{u b} V_{c d}\right) /\left(V_{c b} V_{u d}\right)$. The contribution from the annihilation topology is also absorbed in the quantity $z$, and will be further discussed in Sec. II C.

We can estimate $|C| /(|T|+|C|)$ in two ways.

(A) $r_{B}^{D \pi} \sim A\left(B^{0} \rightarrow \bar{D}^{0} K^{0}\right) / A\left(B^{+} \rightarrow \bar{D}^{0} K^{+}\right)$, applying $\mathrm{SU}(3)$ symmetry, and correcting for the different CKM elements involved.

(B) $r_{B}^{D \pi} \sim A\left(B^{0} \rightarrow \bar{D}^{0} \pi^{0}\right) / A\left(B^{+} \rightarrow \bar{D}^{0} \pi^{+}\right)$, using external estimates for the contribution from $W$-exchange topologies $(E)$ to the decay $B^{0} \rightarrow \bar{D}^{0} \pi^{0}$.
TABLE II. Values of CKM elements used.

\begin{tabular}{ll}
\hline \hline$V_{\mathrm{CKM}}$ & Ref. \\
\hline$\left|V_{u d}\right|=0.97425 \pm 0.00022$ & {$[10]$} \\
$\left|V_{u s}\right|=0.2253 \pm 0.0008$ & {$[10]$} \\
$\left|V_{u b}\right|=(3.72 \pm 0.16) \times 10^{-3}$ & {$[12]$} \\
$\left|V_{c d}\right|=0.225 \pm 0.008$ & {$[10]$} \\
$\left|V_{c s}\right|=0.986 \pm 0.016$ & {$[10]$} \\
$\left|V_{c b}\right|=(41.1 \pm 1.3) \times 10^{-3}$ & {$[10]$} \\
\hline \hline
\end{tabular}

The magnitude of $z$ will be estimated in Sec. III by comparing the result of the amplitude ratio of the decays $B^{+} \rightarrow D^{0} K^{+}, r_{B}^{D K}$, to the measured value by $\mathrm{LHCb}$ [7]. For the numerical values of the CKM elements, we use the values listed in Table II.

\section{A. Estimating $r_{B}^{D \pi}$ from $B^{0} \rightarrow \bar{D}^{0} K^{0}$}

The decays $B \rightarrow D K$ can be used to estimate the contributions of various $B \rightarrow D \pi$ decay topologies, assuming $\mathrm{SU}(3)$ symmetry.

The validity of this assumption was probed by comparing the $D^{(*)} K$ and $D^{(*)} \pi$ decay rates, correcting for differences in phase space, CKM elements, form factors and decay constants [8]. This assures that the decays $B^{0} \rightarrow$ $\bar{D}^{0} K^{0}$ and $B^{+} \rightarrow \bar{D}^{0} K^{+}$can be used to estimate a value for the amplitude ratio, $|C| /|T+C|=\left|C^{\prime}\right| /\left|T^{\prime}+C^{\prime}\right|$, where

$$
\left|\frac{C^{\prime}}{T^{\prime}+C^{\prime}}\right|=\sqrt{\frac{\alpha \mathrm{BR}\left(B^{0} \rightarrow \bar{D}^{0} K^{0}\right)}{\mathrm{BR}\left(B^{+} \rightarrow \bar{D}^{0} K^{+}\right)}} .
$$

The factor $\alpha$ quantifies a correction to the quoted value of $\operatorname{BR}\left(B^{0} \rightarrow \bar{D}^{0} K^{0}\right)$ from the Particle Data Group [10]. The measured branching fraction by the BABAR [13] and Belle [14] collaborations is obtained from the sum over the charge-conjugate final states, and therefore the quoted branching fraction represents the sum of the $B^{0} \rightarrow \bar{D}^{0} K^{0}$ and $B^{0} \rightarrow D^{0} K^{0}$ branching fractions. Recently $\mathrm{LHCb}$ also performed an analysis of the decays $B_{(s)}^{0} \rightarrow \bar{D}^{0} K^{0}[15]$.

The quoted branching fraction can thus be expressed as the sum of the squares of the two color-suppressed tree amplitudes,

$$
\begin{aligned}
\operatorname{BR}\left(B^{0} \rightarrow \stackrel{(-)}{D}^{0} K^{0}\right) & =A\left(B^{0} \rightarrow \bar{D}^{0} K^{0}\right)^{2}+A\left(B^{0} \rightarrow D^{0} K^{0}\right)^{2} \\
& =\left|C^{\prime}\right|^{2}+\left|C^{\prime u b}\right|^{2} \\
& =\left(1+z^{\prime}\left|\frac{V_{u b} V_{c s}}{V_{c b} V_{u s}}\right|^{2}\right) \times\left|C^{\prime}\right|^{2} \\
& =\left(1+0.156 z^{\prime}\right) \times\left|C^{\prime}\right|^{2}
\end{aligned}
$$

where $z^{\prime}$ quantifies the ratio between the hadronic parts of the two color-suppressed tree diagrams proceeding through the $b \rightarrow u$ and $b \rightarrow c$ transitions, 
$\left|C^{\prime u b}\right|=z^{\prime} C^{\prime} \times\left|V_{u b} V_{c s} / V_{c b} V_{u s}\right|$. Hence, we need to correct the quoted branching fraction of the decay $B^{0} \rightarrow \bar{D}^{0} K^{0}$ to yield an estimate of the amplitude of $C^{\prime}$, relative to $\left|T^{\prime}+C^{\prime}\right|$ with $\alpha=1 /\left(1+0.156 z^{\prime}\right)$, to obtain

$$
r_{B}^{D \pi}=\left|\frac{V_{u b} V_{c d}}{V_{c b} V_{u d}}\right| \frac{z^{\prime}}{1+0.156 z^{\prime}} \sqrt{\frac{\mathrm{BR}\left(B^{0} \rightarrow \bar{D}^{0} K^{0}\right)}{\operatorname{BR}\left(B^{+} \rightarrow \bar{D}^{0} K^{+}\right)}} .
$$

\section{B. Estimating $r_{B}^{D \pi}$ from $B^{\mathbf{0}} \rightarrow \bar{D}^{\mathbf{0}} \boldsymbol{\pi}^{\mathbf{0}}$}

A second estimate of $r_{B}^{D \pi}$ can be obtained using the decay $B^{0} \rightarrow \bar{D}^{0} \pi^{0}$. The decay $B^{0} \rightarrow \bar{D}^{0} \pi^{0}$ receives contributions from the color-suppressed tree diagram $(C)$ and from the $W$-exchange diagram $(E)$. The comparison of the $B^{0} \rightarrow \bar{D}^{0} \pi^{0}$ and $B^{+} \rightarrow \bar{D}^{0} \pi^{+}$decay rates gives [8]

$$
\left|\frac{C-E}{T+C}\right|=\sqrt{2} \sqrt{\frac{\mathrm{BR}\left(B^{0} \rightarrow \bar{D}^{0} \pi^{0}\right)}{\operatorname{BR}\left(B^{+} \rightarrow \bar{D}^{0} \pi^{+}\right)}}=0.331 \pm 0.010(\mathrm{BR})
$$

again assuming that CKM elements, form factors, decay constants and phase space factors cancel in the ratio. The uncertainty originates from the uncertainty on the measured branching fractions. The factor $\sqrt{2}$ originates from the isospin decomposition of the neutral pion. Although the branching fraction $\operatorname{BR}\left(B^{0} \rightarrow \bar{D}^{0} \pi^{0}\right)$ is determined as the sum of the $D^{0}$ and $\bar{D}^{0}$ final states, the $b \rightarrow u$ colorsuppressed tree amplitude is negligible compared to the $b \rightarrow c$ amplitude, unlike the situation of Eq. (2).

The color-suppressed tree diagram is expected to dominate the total transition amplitude with respect to the $W$-exchange topology $E$, which is supported by the comparison of the branching fractions of $B^{0} \rightarrow \bar{D}^{0} \pi^{0}$ and $B^{0} \rightarrow \bar{D}^{0} K^{0}[8]$, leading to

$$
\left|\frac{C-E}{C}\right|=0.913 \pm 0.074 \text {. }
$$

To obtain an independent estimate of $r_{B}^{D \pi}$ with respect to Eq. (4), i.e. without reusing information on the branching fraction of $B^{0} \rightarrow \bar{D}^{0} K^{0}$, the size of the $W$-exchange amplitude can be estimated from the decay $B^{0} \rightarrow D_{s}^{-} K^{+}$ [16], resulting in the following value [8]:

$$
\left|\frac{E}{T+C}\right|=0.056 \pm 0.004 \text {. }
$$

Without assuming any value for the relative phase between the $W$-exchange $(E)$ and color-suppressed $(C)$ amplitudes, we assign the full contribution of the $W$-exchange amplitude as uncertainty to the estimate of $|C /| T+C \mid$,

$$
\left|\frac{C}{T+C}\right|=0.331 \pm 0.010(\mathrm{BR}) \pm 0.056(E) .
$$

The resulting expression for $r_{B}^{D \pi}$ then becomes

$$
r_{B}^{D \pi}=\left|\frac{V_{u b} V_{c d}}{V_{c b} V_{u d}}\right| \sqrt{2} z \sqrt{\frac{\mathrm{BR}\left(B^{0} \rightarrow \bar{D}^{0} \pi^{0}\right)}{\mathrm{BR}\left(B^{+} \rightarrow \bar{D}^{0} \pi^{+}\right)}} .
$$

\section{Effect of annihilation topology}

The relative contribution from the annihilation topology with respect to the color-suppressed tree topology for the $B^{+} \rightarrow D^{0} \pi^{+}$amplitude, is estimated using the measured branching fraction of the decay $B^{+} \rightarrow D_{s}^{+} \phi$ [17], relative to the decay $B^{0} \rightarrow D^{0} K^{0}$. At lowest order the $B^{+} \rightarrow D_{s}^{+} \phi$ decay proceeds purely through the annihilation topology.

The estimate of $|A / C|$ for the $B^{+} \rightarrow D^{0} \pi^{+}$case can be directly obtained from the branching ratios, when corrected for by the appropriate CKM elements and decay constants $f_{X}$,

$$
|A / C|=\sqrt{\frac{\mathrm{BR}\left(B^{+} \rightarrow D_{s}^{+} \phi\right)}{\operatorname{BR}\left(B^{0} \rightarrow D^{0} K^{0}\right)}}\left(\frac{V_{c b} V_{u s}}{V_{u b} V_{c s}}\right)\left(\frac{f_{D} f_{K}}{f_{D s} f_{\phi}}\right) \sim 0.25
$$

with a large uncertainty from the branching fraction measurement of $B^{+} \rightarrow D_{s}^{+} \phi$; see Table I. It is also noted that the branching fraction $\operatorname{BR}\left(B^{+} \rightarrow \bar{D}^{0} D_{s}^{+}\right)$deviates from $\operatorname{BR}\left(B^{0} \rightarrow D^{-} D_{s}^{+}\right)$, where the main difference is expected to arise from the annihilation contribution [18]. Possible contributions to these final states from rescattering processes are discussed in Ref. [19]. The relative phase between the annihilation and color-suppressed tree topology is unknown, so the annihilation contribution can enhance or reduce the value of $r_{B}^{D \pi}$. Assuming SU(3) symmetry, this contribution is expected to be equal in the $B^{+} \rightarrow D^{0} K^{+}$system, and will thus be accounted for in the determination of $z$ from $r_{B}^{D K}$ in the next section.

\section{CORRECTION USING $r_{B}^{D K}$}

To quantify the ratio $z$ between the hadronic parts of the $b \rightarrow u$ and $b \rightarrow c$ color-suppressed tree diagrams, $C^{\prime u b}=z^{\prime} C^{\prime} \times\left(V_{u b} V_{c s} / V_{c b} V_{u s}\right)$, an estimate for $r_{B}^{D K}$ can be obtained in a similar way, and be compared to the fitted value for $r_{B}^{D K}$ from the LHCb fit [7]. The quantity $z$ also contains the correction due to contributions from the annihilation topology; see Fig. 1. We obtain the following expression for $r_{B}^{D K}$ :

$$
r_{B}^{D K}=\left|\frac{V_{u b} V_{c s}}{V_{c b} V_{u s}}\right| \frac{z^{\prime}}{1+0.156 z^{\prime}} \sqrt{\frac{\mathrm{BR}\left(B^{0} \rightarrow \bar{D}^{0} K^{0}\right)}{\mathrm{BR}\left(B^{+} \rightarrow \bar{D}^{0} K^{+}\right)}},
$$

which differs from Eq. (4) by the different CKM elements involved. Inserting the value for $r_{B}^{D K}$ obtained from the 
LHCb fit, $r_{B}^{D K}=0.101 \pm 0.006$ [7], the following estimate for the ratio of the hadronic parts of the color-suppressed amplitudes is obtained:

$$
\frac{z^{\prime}}{1+0.156 z^{\prime}}=0.68 \pm 0.05 \Rightarrow z^{\prime}=0.76 \pm 0.07 \text {. }
$$

The fact that the value of $z^{\prime}$ is close to unity, indicates that the hadronic parts of the two color-suppressed tree diagrams are of similar magnitude, in particular if the annihilation topology negatively interferes with the colorsuppressed tree topology, i.e. if the relative strong phase is close to $180^{\circ}$, which would lead to a value $z^{\prime} \sim 0.75$. We assume that the deviation from unity is equal for the $D \pi$ case, with an uncertainty of $10 \%$ from $\mathrm{SU}(3)$ symmetry breaking effects, $z=0.76 \pm 0.07(\mathrm{BR}) \pm 0.02(\mathrm{SU}(3))$.

Inserting the numerical values in Eq. (4) and Eq. (9) leads to the following estimates of $r_{B}^{D \pi}$ :

$$
\begin{aligned}
r_{B}^{D \pi}\left(D^{0} K^{0}\right)= & 0.0053 \pm 0.0002\left(\mathrm{~V}_{\mathrm{CKM}}\right) \pm 0.0004(\mathrm{BR}) \\
& \pm 0.0005(\mathrm{SU}(3)) \\
r_{B}^{D \pi}\left(D^{0} \pi^{0}\right)= & 0.0053 \pm 0.0002\left(\mathrm{~V}_{\mathrm{CKM}}\right) \pm 0.0002(\mathrm{BR}) \\
& \pm 0.0009(\mathrm{E}) \pm 0.0005(z)
\end{aligned}
$$

which are in good agreement, albeit with a large uncertainty from the $W$-exchange contribution to the $B^{0} \rightarrow \bar{D}^{0} \pi^{0}$ decay rate. The agreement shows the internal consistency of the approach presented here. An additional 10\% uncertainty from SU(3) symmetry is assumed in the estimate of Eq. (11), based on the agreement of the relative contributions of the various decay topologies to the $B \rightarrow$ $D K$ and $B \rightarrow D \pi$ decays [8]. Given the correlated systematic uncertainties between the two results, the following combined estimate is obtained:

$$
r_{B}^{D \pi}=0.0053 \pm 0.0007
$$

\section{CONCLUSIONS}

The estimate for the value of the amplitude ratio $r_{B}^{D \pi}$ that is presented here provides a valuable input to the discussion of the measurement of $r_{B}^{D \pi}$ at LHCb. The actual measurement of $r_{B}^{D \pi}$ can be achieved either by a combination of indirect measurements, as presented in Refs. [6,7], or by direct measurement using semileptonic decays of the form $B^{+} \rightarrow D^{0} \pi^{+}$, where $D^{0} \rightarrow K^{-} \mu^{+} \nu_{\mu}$, and the charge of the kaon and muon can unambiguously tag the $D^{0}$ flavor. Future determinations of $r_{B}^{D \pi}$ can be compared to the estimate presented here, to assess the validity of the assumptions on rescattering and $\mathrm{SU}(3)$ symmetry as used in this paper. The LHCb Collaboration foresees accumulating a 4 times larger data set by the end of 2018, and a 5 times smaller uncertainty at the end of the LHCb upgrade, which will result in an experimental uncertainty of the measured value of $r_{B}^{D \pi}$ that is smaller than the one presented here.

\section{ACKNOWLEDGMENTS}

We would like to thank Robert Fleischer, Vincenzo Vagnoni and Greg Ciezarek for many valuable discussions.
[1] J. Charles et al. (CKMfitter Collaboration), Current status of the Standard Model CKM fit and constraints on $\Delta F=2$ New Physics, Phys. Rev. D 91, 073007 (2015).

[2] M. Bona et al. (UTfit Collaboration), An improved Standard Model prediction of $\operatorname{BR}(B \rightarrow \tau \nu)$ and its implications for New Physics, Phys. Lett. B 687, 61 (2010).

[3] P. del Amo Sanchez et al. (BABAR Collaboration), Search for $b \rightarrow u$ transitions in $B^{-} \rightarrow D K^{-}$and $D^{*} K^{-}$decays, Phys. Rev. D 82, 072006 (2010).

[4] Y. Horii et al. (Belle Collaboration), Evidence for the Suppressed Decay $B^{-} \rightarrow D K^{-}, D \rightarrow K^{+} \pi^{-}$, Phys. Rev. Lett. 106, 231803 (2011).

[5] R. Aaij et al. (LHCb Collaboration), A measurement of the CKM angle $\gamma$ from a combination of $B^{ \pm} \rightarrow D h^{ \pm}$analyses, Phys. Lett. B 726, 151 (2013).

[6] R. Aaij et al. (LHCb Collaboration), Report No. LHCbCONF-2014-004, http://inspirehep.net/record/1388239? $\ln =\mathrm{en}$.

[7] R. Aaij et al. (LHCb Collaboration), Report No. LHCbPAPER-2016-032.
[8] R. Fleischer, N. Serra, and N. Tuning, Tests of factorization and $S U(3)$ relations in $B$ decays into heavy-light final states, Phys. Rev. D 83, 014017 (2011).

[9] K. De Bruyn, R. Fleischer, R. Knegjens, M. Merk, M. Schiller, and N. Tuning, Exploring $B_{s} \rightarrow D_{s}^{(*) \pm} K^{\mp}$ decays in the presence of a sizable width difference $\Delta \Gamma_{s}$, Nucl. Phys. B868, 351 (2013).

[10] K. A. Olive et al. (Particle Data Group), Review of Particle Physics, Chin. Phys. C 38, 090001 (2014) and 2015 update.

[11] M. Beneke, G. Buchalla, M. Neubert, and C. Sachrajda, QCD factorization for exclusive, non-leptonic B meson decays: General arguments and the case of heavy-light final states, Nucl. Phys. B591, 313 (2000).

[12] J. A. Bailey et al. (Fermilab Lattice and MILC Collaborations), $\left|V_{u b}\right|$ from $B \rightarrow \pi \ell \nu$ decays and $(2+1)$-flavor lattice QCD, Phys. Rev. D 92, 014024 (2015).

[13] B. Aubert et al. (BABAR Collaboration), Measurement of $\bar{B}^{0} \rightarrow D^{(*) 0} \bar{K}^{(*) 0}$ branching fractions, Phys. Rev. D 74, 031101 (2006). 
ESTIMATING $r_{B}^{D \pi}$ AS AN INPUT TO THE ...

[14] P. Krokovny et al. (Belle Collaboration), Observation of $\bar{B}^{0} \rightarrow D^{0} \bar{K}^{0}$ and $\bar{B}^{0} \rightarrow D^{0} \bar{K}^{* 0}$ Decays, Phys. Rev. Lett. 90, 141802 (2003).

[15] R. Aaij et al. (LHCb Collaboration), Observation of $\quad B_{s}^{0} \rightarrow \bar{D}^{0} K_{S}^{0}$, Phys. Rev. Lett. 116, 161802 (2016).

[16] R. Aaij et al. (LHCb Collaboration), Determination of the branching fractions of $B_{s}^{0} \rightarrow D_{s}^{\mp} K^{ \pm}$and $B^{0} \rightarrow D_{s}^{-} K^{+}, \mathrm{J}$. High Energy Phys. 05 (2015) 019.
PHYSICAL REVIEW D 94, 054021 (2016)

[17] R. Aaij et al. (LHCb Collaboration), First evidence for the annihilation decay mode $B^{+} \rightarrow D_{s}^{+} \phi$, J. High Energy Phys. 02 (2013) 043.

[18] L. Bel, K. De Bruyn, R. Fleischer, M. Mulder, and N. Tuning, Anatomy of $B \rightarrow D \bar{D}$ decays, J. High Energy Phys. 07 (2015) 108.

[19] M. Gronau, D. London, and J. L. Rosner, Rescattering contributions to rare $B$-meson decays, Phys. Rev. D 87, 036008 (2013). 